Research Article

\title{
Research on the Impact of Marine Environment on ICPT Transmission Efficiency
}

\author{
Da Li $(\mathbb{D}$, Wei Gao $\mathbb{D}$, Xusheng Wu $(\mathbb{D}$, Jianxin Gao $\mathbb{D}$, and Shenqin Yang $\mathbb{C}$ \\ College of Electrical Engineering, Naval Engineering University, Wuhan 43003, China \\ Correspondence should be addressed to Wei Gao; depkin@163.com, Xusheng Wu; wuxusheng_hg@163.com, Jianxin Gao; \\ gaojianxin_cn@163.com, and Shenqin Yang; 1171146351@qq.com
}

Received 21 April 2020; Revised 24 September 2020; Accepted 30 September 2020; Published 30 October 2020

Academic Editor: Ding-Bing Lin

Copyright $\odot 2020 \mathrm{Da}$ Li et al. This is an open access article distributed under the Creative Commons Attribution License, which permits unrestricted use, distribution, and reproduction in any medium, provided the original work is properly cited.

ICPT is one of the most influential solutions in the field of wireless power transmission, but it is also very susceptible to the working environment. The complicated marine environment has a great influence on the performance of ICPT. In the deep-sea high-pressure environment, due to the piezomagnetic effect, the magnetic core in the coupling structure will suddenly change the permeability, and the coupling coefficient, self-inductance, and other parameters will also change accordingly. At the same time, changes in ocean currents in the ocean will cause the ICPT coils to be misaligned, thereby affecting the magnetic field distribution between the coils and the transmission efficiency of the system. In order to provide theoretical support for optimum design of ICPT system in ocean environment, the influence of core performance changes and ICPT coil misalignment on system transmission efficiency is studied.

\section{Introduction}

At present, wireless power transmission has been widely used in the consumer electronics, transportation, industry, and some special application scenarios [1-5]. However, with the development of marine equipment electromechanical, the traditional wet plug interface and wire power transmission cannot meet the energy supply of unmanned submersibles, unmanned ships, and other marine equipment. The wireless power transmission has realized the source and charging equipment completely isolated. It has solved the problems of low security, high requirements for removable seals, frequent plugging, etc. Wireless power transmission has unique advantages in deep sea extreme environments and special conditions $[6,7]$.

At this stage, the research on wireless power transmission technology on the road is rapid [8], especially in the circuit theory research of wireless power transmission [1-5]. However, the research on wireless power transmission for the marine environment is still relatively small. There are more factors in the marine than on the terrestrial that affect the transmission power of the system. Seawater has good electrical conductivity; the transmission of high-frequency electromagnetic fields in seawater will cause eddy current loss and reduce the transmission efficiency of wireless power transmission [14]. Zhang et al. established a mathematical model of magnetic resonance power transmission system in the air and derived the mathematical expression of eddy current loss based on Maxwell's equations. The relationship between the mutual inductance with air medium, eddy current loss and resonance frequency, coil radius, dielectric conductivity, dielectric thickness, etc. were studied [15]; Askari et al. set up a four-coil resonance comparison test between air and seawater. The results showed that the power loss of seawater increases with the increase of resonance frequency, while the power loss increases obviously when the resonance frequency is above $20 \mathrm{kHz}$ [16]; Shizuno et al. designed a wireless power transmission device suitable for seawater and achieved a transmission distance of $50 \mathrm{~mm}$. The system transmission efficiency is $50 \%$, and it is found that eddy current loss is the main form of energy loss [17]. Not only does the eddy current loss exist in the marine but also the huge water pressure, ocean current disturbance, seawater composition, and water temperature in the deep sea will affect the performance of the wireless power transmission. Under the deep-sea environment, there is huge 
water pressure, which not only has higher requirements for the packaging of electric power transmission equipment but also reduces the permeability and affects the transmission performance of the system. The high pressure causes the magnetic core of the coupling mechanism to have a "piezomagnetic effect." The ocean current is complicated in the deep sea; the impact of the water current can easily cause the loosely coupled coils to be misaligned, changing the coupling state of the system and reducing the stability of the system, such as seawater temperature, salinity, and microorganisms, could cause changes in system parameters and affect the performance of wireless power transmission.

Compared with air, the variables that affect ocean transmission efficiency are more complex. This paper studies the influence of seawater as a special transmission medium on transmission performance from the perspective of seawater pressure, coil misalignment, and eddy current loss and provides theoretical support for the optimization of underwater ICPT (Inductively Coupled Power Transfer) efficiency.

\section{Influencing Factors of Marine on ICPT}

2.1. Influence of Seawater Pressure on ICPT. High pressure is the typical characteristic of deep sea. Mechanical and electrical equipment will withstand the pressure from seawater when working in deep sea. The pressure of seawater on working equipment increases linearly with the increase of seawater depth, as shown in Figure 1.

Soft ferrite with high permeability is usually added to the coupling coil of ICPT to improve its quality factor. Under the effect of external force, the material will produce internal stress, which will lead to the change of permeability material. The phenomenon of the material's permeability changing under the external force is usually called the piezoelectric effect. The initial permeability of the material in reversible magnetization under external force [18]

$$
\mu_{i}=\frac{2 \mu_{0} M_{S}^{2} l}{3 \pi^{2} \delta \lambda_{S} \sigma}+1 .
$$

$\sigma$ is the internal stress of material, which will change under the stress of external force; $\lambda_{S}$ and $M_{S}$ are the magnetostrictive coefficient and saturation magnetization of the material; and $l$ and $\delta$ represent the width and thickness of the internal magnetic domain wall of the material. Since the permeability $\mu$ of ferrite in soft magnetic materials is much greater than 1, the piezomagnetic effect of such materials under external force can be expressed as

$$
\frac{\Delta \mu_{i}}{\mu_{i}}=\frac{\left(1-\mu_{i}\right)}{\mu_{i}} \cdot \frac{\Delta \sigma}{\sigma} \approx-\frac{\Delta \sigma}{\sigma}
$$

It can be seen from formula (2) that the permeability of the material will decrease with the increase of stress on the material. The permeability of ferrite directly affects the selfinductance and mutual inductance of ICPT coil with ferrite core. When the coil is located in the deep sea where there is high pressure, the reduction of inductance and mutual inductance of the coupling coil will directly affect the coupling degree of the coil and even lead to the resonance network

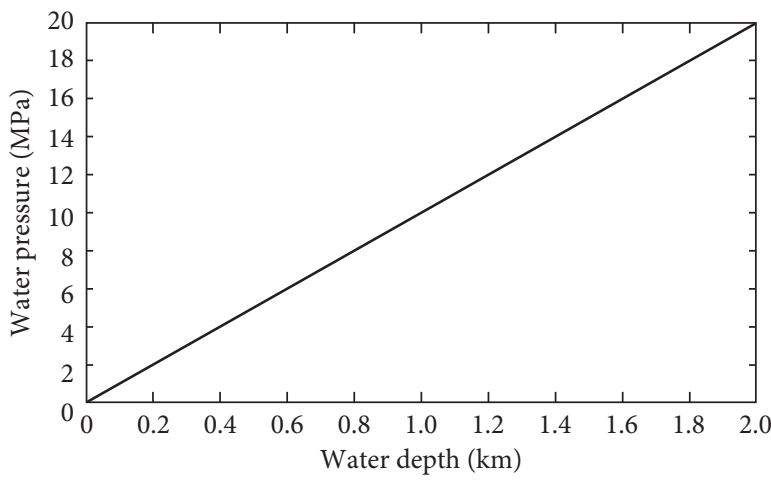

FiguRE 1: Seawater pressure with depth.

detuning, which will further affect the transmission efficiency of ICPT.

This article uses PC40 ferrite which is commonly used in wireless power transfer. This material belongs to $\mathrm{Ni}-\mathrm{Zn}$ series ferrite. The piezomagnetic curve of this type of ferrite is shown in Figure 2 [18]. It can be seen from the figure that when the pressure is 50 bar (the system is at a depth of $5 \mathrm{~km}$ ), and the ferrite permeability reduction rate can reach $40 \%$. Combining with the data in the figure, the self-inductance and mutual inductance of the coupling coil at the depth of $0 \sim 5 \mathrm{~km}$ are simulated by using ANSYS. It is not difficult to find that the self-inductances of the primary and secondary coils under standard atmospheric pressure in the model are $246 \mu \mathrm{H}$ and $88 \mu \mathrm{H}$, respectively. The length, width, and height of the coupler are $370 \mathrm{~mm} \times 320 \mathrm{~mm} \times 15 \mathrm{~mm}$, and the simulation interface is shown in Figure 3.

From the curve of coil coupling coefficient with water depth, it can be seen from Figure 4(a) that the gaps between primary coil and secondary coil are $10 \mathrm{~mm}, 1 \mathrm{~mm}$, and $0.5 \mathrm{~mm}$, respectively. Under the same coil spacing, the coupling coefficient of the primary coil and secondary coil is not affected by water depth. From the normalized self-inductance curve of the coil shown in Figure 4(b), the coil gap affects the change rate of the coil self-inductance value. The coil gap is greater than $1 \mathrm{~mm}$, the water depth has little effect on the self-inductance value of the coil, but with the increase of the coil gap, the water depth has little effect. In this case, the self-inductance of the coil is reduced by $13 \%$ compared with the vacuum environment.

2.2. Effect of Coil Position. In the process of underwater wireless power transfer, electrical equipment such as AUV and charging base station are usually not fixed firmly. Under the impact of underwater currents, the secondary coils on the electrical equipment are easy to shift and tilt, which affects the stability of system transmission.

2.2.1. Coil Horizontal Offset. In Figure 5, the width of a single-turn coil is represented by $W$ and the turn distance is represented by $d$. The length and width of the outermost coil are $2 l_{1}$ and $2 r_{1}$. The length and width of the $i$ th coil from outside to inside are defined as $2 L_{i}$ and $2 r_{i}$; then, $L_{i}$ and RI can be expressed as 


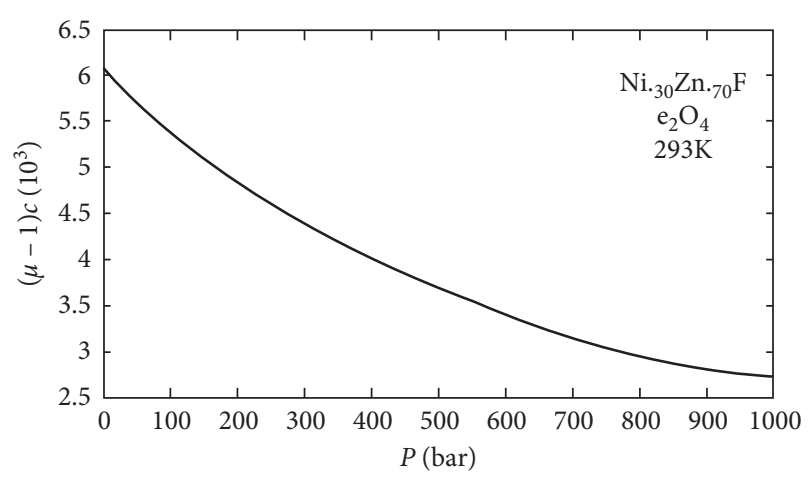

FIgure 2: Piezomagnetic curve of Ni-Zn ferrite.

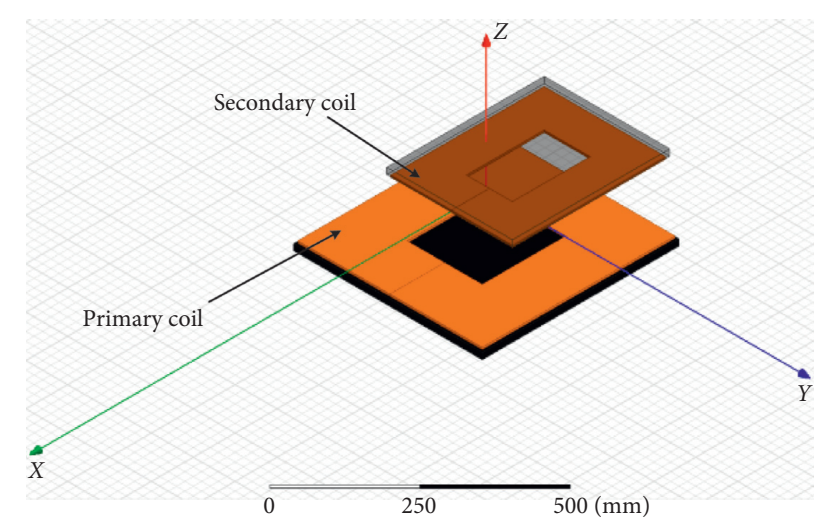

Figure 3: Simulation model of coupling coil.

$$
\left\{\begin{array}{l}
l_{i}=l_{1}-(i-1)(w+d), \\
r_{i}=r_{1}-(i-1)(w+d) .
\end{array}\right.
$$

Figure 6 shows the relative position of the ith primary coil and the $j$ th secondary coil when the secondary coil is horizontally offset. The length and width of the two coils are $2 l_{P i}, 2 r_{P i}, 2 l_{S i}$, and $2 r_{S i}$, respectively. In this diagram, the secondary coil is offset along the positive direction of the $Y$ axis, the offset is $\Delta$, and the gap between the two coils is $D$. A changing magnetic field is excited around the coil when the alternating current $I$ is applied to the primary coil. The magnetic flux in the secondary coil is defined by the magnetic field which is generated by the current I of the primary coil. For $\Phi i j$, the mutual inductance between the two coils can be expressed as shown in equation (3):

$$
M_{i j}=\frac{\phi_{i j}}{I} .
$$

The magnetic flux $\Phi_{i j}$ is calculated as

$$
\phi_{i j}=\int_{S_{j}} B_{i Z} \mathrm{~d} S_{j}=\int_{S_{j}} B \cdot \cos \theta \mathrm{d} S_{j} .
$$

In formula (5), $S_{j}$ represents the area surrounded by the $j$ th secondary coil, $B_{i z}$ is the component of magnetic induction intensity generated by the ith primary coil perpendicular to the plane of the secondary coil, and $\theta$ is the angle between the normal lines of the plane where the magnetic induction coil is located.

Suppose that the $K$ coordinate of any point in the rectangle surrounded by the secondary coil $I$ is $K(x, y, d)$. According to Biot Savart law, the primary coil passes through alternating at the current $I$, the magnitude of the magnetic induction $b c$ generated at the point $K$ of the lead segment $b c$ is

$$
B_{b c}=\frac{\mu_{0} I}{4 \pi} \cdot \frac{1}{\sqrt{\left(l_{p i}-y\right)^{2}+D^{2}}} \cdot\left[\frac{r_{p i}+x}{\sqrt{\left(l_{p i}-y\right)^{2}+D^{2}+\left(r_{p i}+x\right)^{2}}}+\frac{r_{p i}-x}{\sqrt{\left(l_{p i}-y\right)^{2}+D^{2}+\left(r_{p i}-x\right)^{2}}}\right] .
$$

The cosine of angle $\theta$ can be expressed as

$$
\cos \theta=\frac{l_{p i}-y}{\sqrt{\left(l_{p i}-y\right)^{2}+D^{2}}}
$$

The component of the magnetic induction intensity along the plane perpendicular of the secondary coil is

$$
B_{b c_{-} Z}=\frac{\mu_{o} I}{4 \pi} \cdot \frac{l_{p i}-y}{\left(l_{p i}-y\right)^{2}+D^{2}} \cdot\left[\frac{r_{p i}+x}{\sqrt{\left(l_{p i}-y\right)^{2}+D^{2}+\left(r_{p i}+x\right)^{2}}}+\frac{r_{p i}-x}{\left(l_{p i}-y\right)^{2}+D^{2}+\left(r_{p i}-x\right)^{2}}\right] .
$$

Therefore, the magnetic flux generated by the guide line $b c$ in the surface $s_{j}$ can be expressed as

$$
\phi_{b c_{-} s j}=\int_{-l_{s j+\Delta}}^{l_{s j}+\Delta} \mathrm{d} y \int_{-r_{s j}}^{r_{s j}} B_{b c_{-} z} \mathrm{~d} x \text {. }
$$




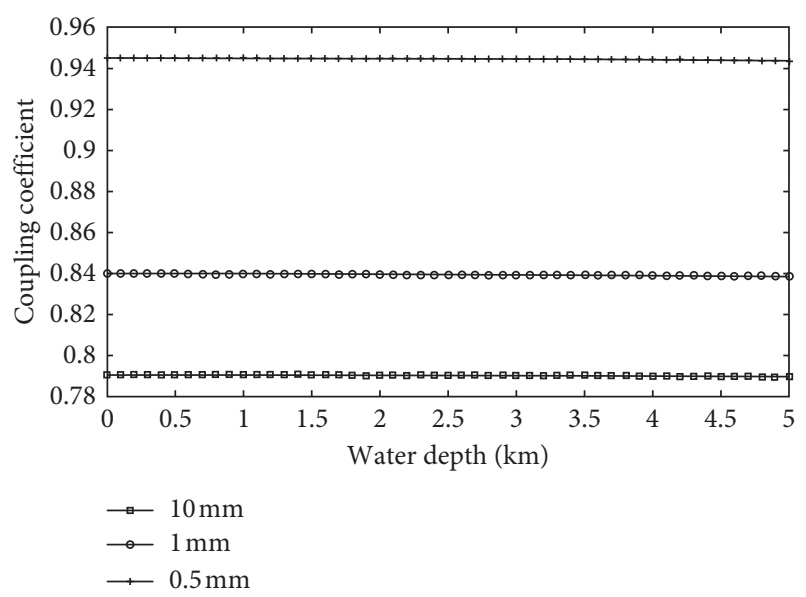

(a)

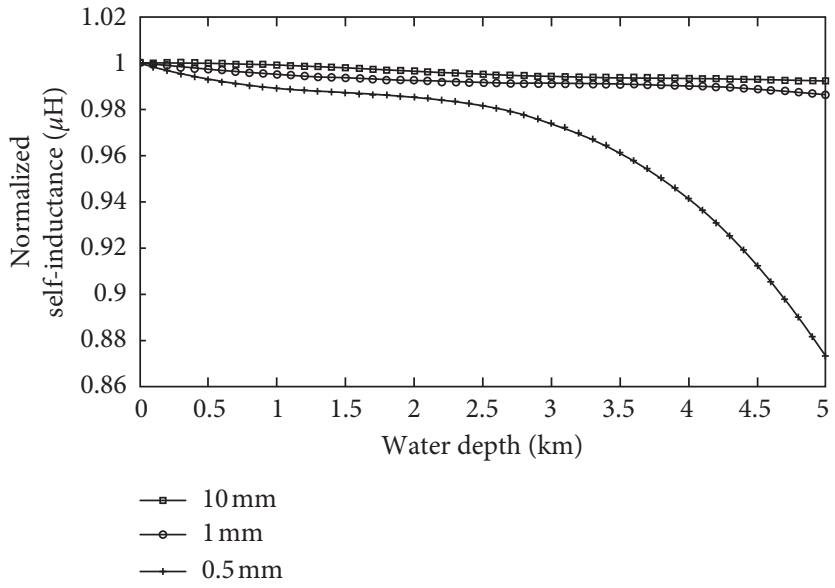

(b)

FIGURE 4: Simulation results of coil self-inductance and mutual inductance. (a) Coupling coefficient with water depth. (b) Self-induction with water depth.

By substituting equation (9) into the above double integral,

$$
\phi_{b c_{s j}} \frac{\mu_{0} I}{2 \pi} \cdot\left[\begin{array}{c}
\sqrt{\left(l_{p i}+l_{s j}-\Delta\right)^{2}+D^{2}+\left(r_{p i}+r_{s j}\right)^{2}}-\left(r_{p i}+r_{s j}\right) \\
\cdot \operatorname{arctanh} \frac{r_{p i}+r_{s j}}{\sqrt{\left(l_{p i}+l_{s j}-\Delta\right)^{2}+D^{2}+\left(r_{p i}+r_{s j}\right)^{2}}} \\
-\sqrt{\left(l_{p i}+l_{s j}-\Delta\right)^{2}+D^{2}+\left(r_{p i}-r_{s j}\right)^{2}}+\left(r_{p i}-r_{s j}\right) \\
\cdot \operatorname{arctanh} \frac{r_{p i}-r_{s j}}{\sqrt{\left(l_{p i}+l_{s j}-\Delta\right)^{2}+D^{2}+\left(r_{p i}+r_{s j}\right)^{2}}} \\
-\sqrt{\left(l_{p i}-l_{s j}-\Delta\right)^{2}+D^{2}+\left(r_{p i}+r_{s j}\right)^{2}}+\left(r_{p i}+r_{s j}\right) \\
\cdot \operatorname{arctanh} \frac{r_{p i}+r_{s j}}{\sqrt{\left(l_{p i}-l_{s j}-\Delta\right)^{2}+D^{2}+\left(r_{p i}+r_{s j}\right)^{2}}} \\
\cdot \operatorname{arctanh} \frac{r_{p i}-r_{s j}}{\sqrt{\left(l_{p i}-l_{s j}-\Delta\right)^{2}+D^{2}+\left(r_{p i}-r_{s j}\right)^{2}}}
\end{array}\right] .
$$

The analysis can be carried out in the same way while the secondary coil is horizontally shifted by $\Delta$ in the positive direction of the $X$-axis. At this time, the magnetic flux generated by the wire end $b c$ in the plane $S_{j}$ is 


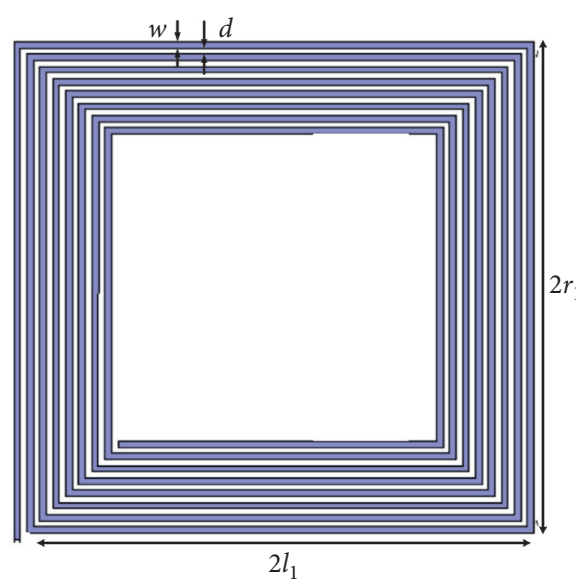

FIGURE 5: ICPT transfer coil winding.

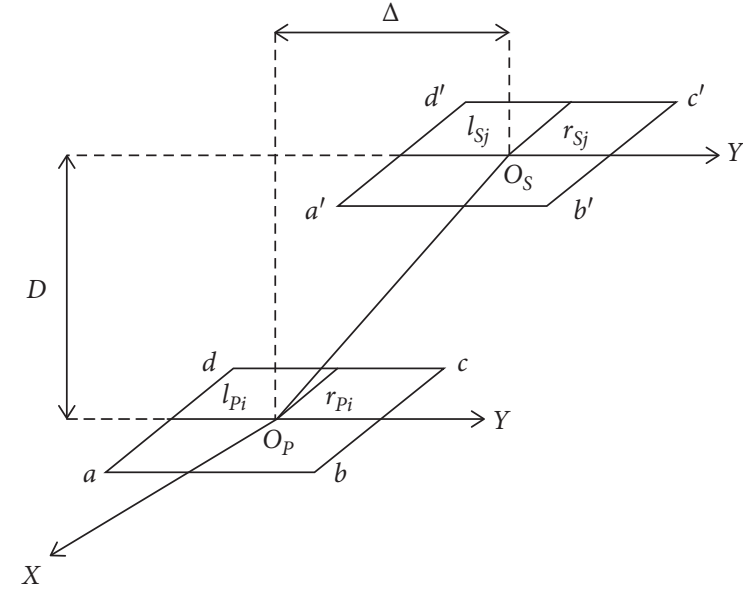

FIgURE 6: Relative position of coil horizontal offset.

$$
\phi_{b c_{s j}^{\prime}}^{\prime} \frac{\mu_{0} I}{2 \pi} \cdot\left[\begin{array}{c}
\sqrt{\left(l_{p i}+l_{s j}\right)^{2}+D^{2}+\left(r_{p i}+r_{s j}-\Delta\right)^{2}}-\left(r_{p i}+r_{s j}-\Delta\right) \\
\cdot \operatorname{arctanh} \frac{r_{p i}+r_{s j}-\Delta}{\sqrt{\left(l_{p i}+l_{s j}\right)^{2}+D^{2}+\left(r_{p i}+r_{s j}-\Delta\right)^{2}}} \\
-\sqrt{\left(l_{p i}+l_{s j}\right)^{2}+D^{2}+\left(r_{p i}-r_{s j}+\Delta\right)^{2}}+\left(r_{p i}-r_{s j}+\Delta\right) \\
\cdot \operatorname{arctanh} \frac{r_{p i}-r_{s j}+\Delta}{\sqrt{\left(l_{p i}+l_{s j}\right)^{2}+D^{2}+\left(r_{p i}-r_{s j}+\Delta\right)^{2}}} \\
\cdot \operatorname{arctanh} \frac{r_{p i}^{2}+r_{s j}-\Delta}{\sqrt{\left(l_{p i}-l_{s j}\right)^{2}+D^{2}+\left(r_{p i}+r_{s j}-\Delta\right)^{2}}} \\
\cdot \operatorname{arctanh} \frac{r_{p i}-r_{s j}+\Delta}{\sqrt{\left(l_{p i}-l_{s j}\right)^{2}+D^{2}+\left(r_{p i}-r_{s j}+\Delta\right)^{2}}+\left(r_{p i}+r_{s j}-\Delta\right)}
\end{array}\right]
$$

In the same way, the magnetic fluxes of the wire segments $c d$, $d a$, and $a b$ in the secondary coil can be obtained, respectively, and expressed as $\Phi_{c d_{-} S j}, \Phi_{d a \_s j}$, and $\Phi_{a b_{-} s j}$. Therefore, when the AC current $I$ flows through the coil $i$, the total magnetic flux through the coil $j$ is

$$
\phi_{i j}=\phi_{a b_{-} s j}+\phi_{b c_{-} s j}+\phi_{c d_{-} s j}+\phi_{d a_{-} s j} .
$$

According to formula (4), the turns of primary coil and secondary coil are $m$ and $n$, respectively. The mutual inductance of the two coils is

$$
M=\sum_{i=1}^{m} \sum_{j=1}^{n} \frac{\phi_{i j}}{I} .
$$

2.2.2. Coil Angle Tilt. The coil angle is inclined while the secondary coil is tilted, the relative position of the $i$ th primary coil and the $j$ th secondary coil are shown in Figure 7. The secondary coil shown is tilted clockwise around the rotor. In $X$-axis, inclination angle is $\gamma$. Because the coil is axisymmetric and the magnetic field generated by the primary coil will not interact with the secondary coil when the 


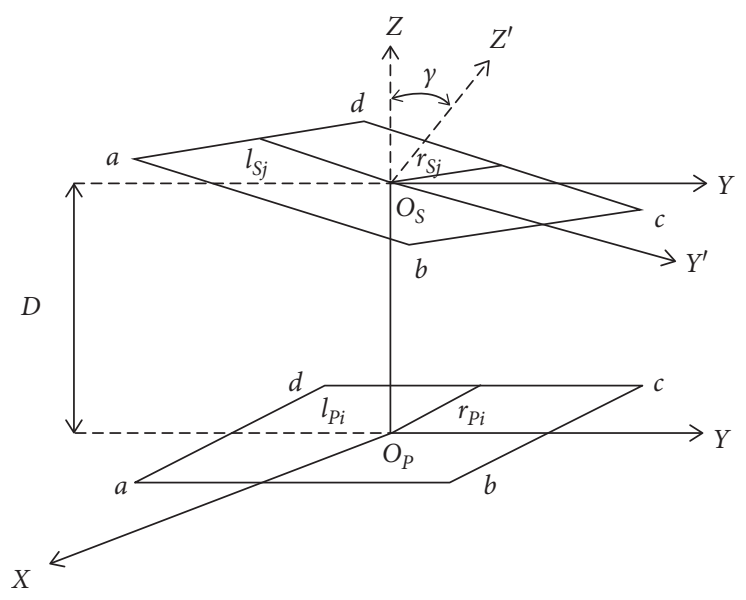

Figure 7: Relative position of coil tilt.

tilt angle is $90^{\circ}$. Therefore, the angle tilt range analyzed here is $0^{\circ} \leq \gamma<90^{\circ}$.

The normal of the inclined secondary coil plane is parallel, and the $K$ coordinate of any point on the right side of $X$-axis in the secondary coil can be expressed as $(x, y$, $d-y \tan \gamma$ ). The magnetic induction intensity is

$$
B_{b c}=\frac{\mu_{0} I}{4 \pi} \cdot \frac{1}{\sqrt{\left(l_{p i}-y\right)^{2}+(D-y \tan \gamma)^{2}}} \cdot\left[\begin{array}{c}
\frac{r_{p i}+x}{\sqrt{\left(l_{p i}-y\right)^{2}+(D-y \tan \gamma)^{2}+\left(r_{p i}+x\right)^{2}}} \\
+\frac{r_{p i}-x}{\sqrt{\left(l_{p i}-y\right)^{2}+(D-y \tan \gamma)^{2}+\left(r_{p i}-x\right)^{2}}}
\end{array}\right] .
$$

The component of the magnetic induction intensity in the $Z^{\prime}$ direction is

$$
B_{b c_{-} Z^{\prime}}=\frac{\mu_{0} I}{4 \pi} \cdot \frac{l_{p i}-y}{\sqrt{\left(l_{p i}-y\right)^{2}+(D-y \tan \gamma)^{2}}} \cdot \cos \gamma \cdot\left[\begin{array}{c}
\frac{r_{p i}+x}{\sqrt{\left(l_{p i}-y\right)^{2}+(D-y \tan \gamma)^{2}+\left(r_{p i}+x\right)^{2}}} \\
\left.+\frac{r_{p i}-x}{\sqrt{\left(l_{p i}-y\right)^{2}+(D-y \tan \gamma)^{2}+\left(r_{p i}-x\right)^{2}}}\right] \\
\phi_{b c_{-} s j}=\int_{-l_{s j} \cos \gamma}^{l_{s j} \sin \gamma} \mathrm{d} y \int_{-r_{s j}}^{r_{s j}} B_{b c_{-} Z^{\prime}} \mathrm{d} x .
\end{array}\right.
$$


TABle 1: Coil parameters.

\begin{tabular}{lc}
\hline Parameter & Value \\
\hline Primary coil self-inductance $\left(L_{P} / \mu \mathrm{H}\right)$ & 246 \\
Secondary coil self-inductance $\left(L_{S} / \mu \mathrm{H}\right)$ & 88 \\
Primary coil turns $m$ (turn) & 18 \\
Secondary coil turns $n$ (turn) & 13 \\
Turn pitch $d(\mathrm{~mm})$ & 7.2 \\
Single-turn wire diameter $w(\mathrm{~mm})$ & 5 \\
Transmission gap $D(\mathrm{~mm})$ & 30 \\
First turn primary coil side length $2 l_{P 1}, 2 r_{P 1}(\mathrm{~mm})$ & 460,440 \\
First turn secondary coil side length $2 l_{S 1}, 2 r_{S 1}(\mathrm{~mm})$ & 370,290 \\
Excitation current amplitude $I(\mathrm{~A})$ & 15 \\
Excitation current frequency $f(\mathrm{kHz})$ & 85 \\
\hline
\end{tabular}

Substituting equation (15) into the above double integral,

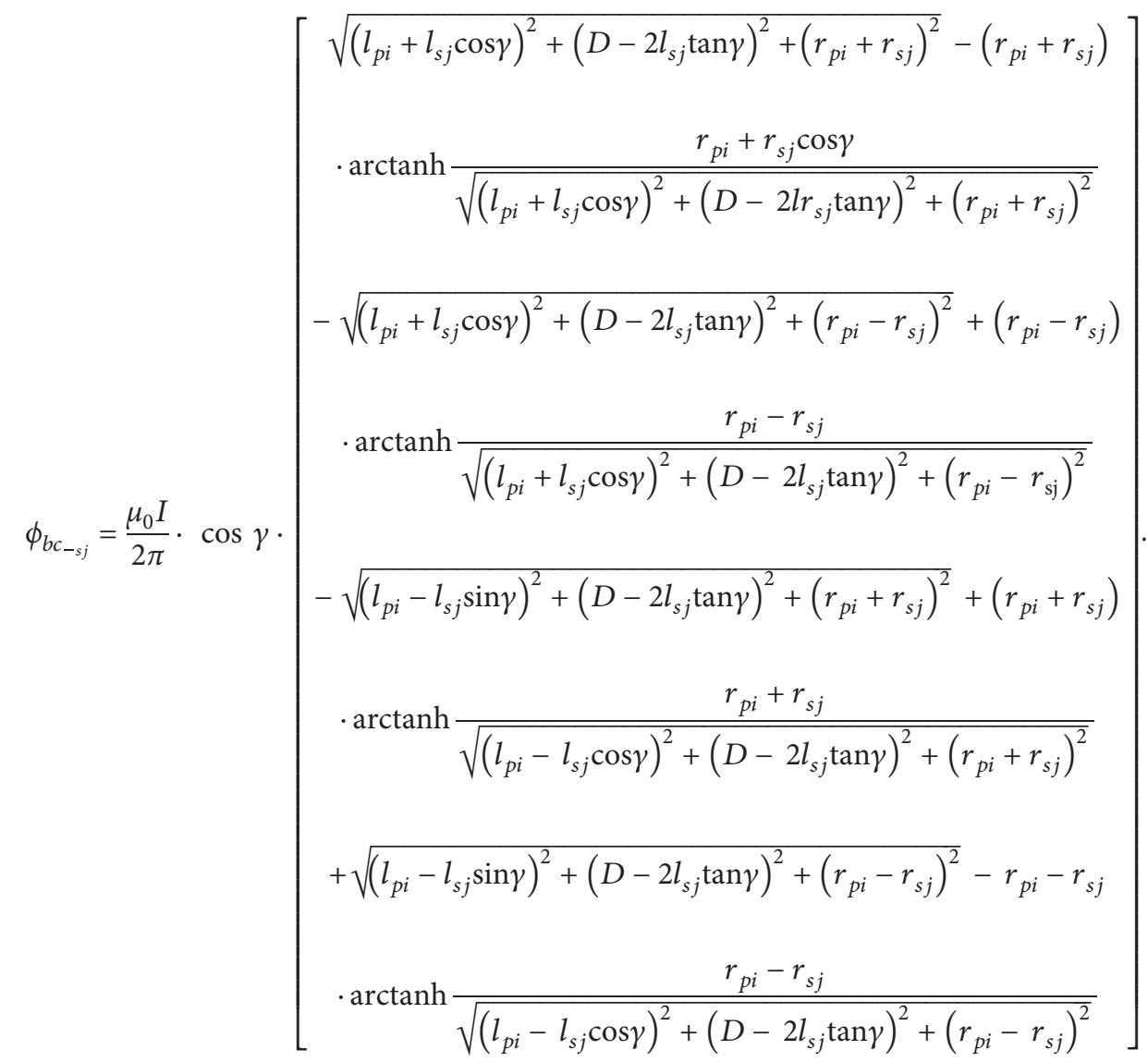


Similarly, the secondary coil is tilted by an angle of $\gamma$ about the $Y$-axis, the magnetic flux generated in the plane $S_{j}$ by the wire segment $b c$ is shown by

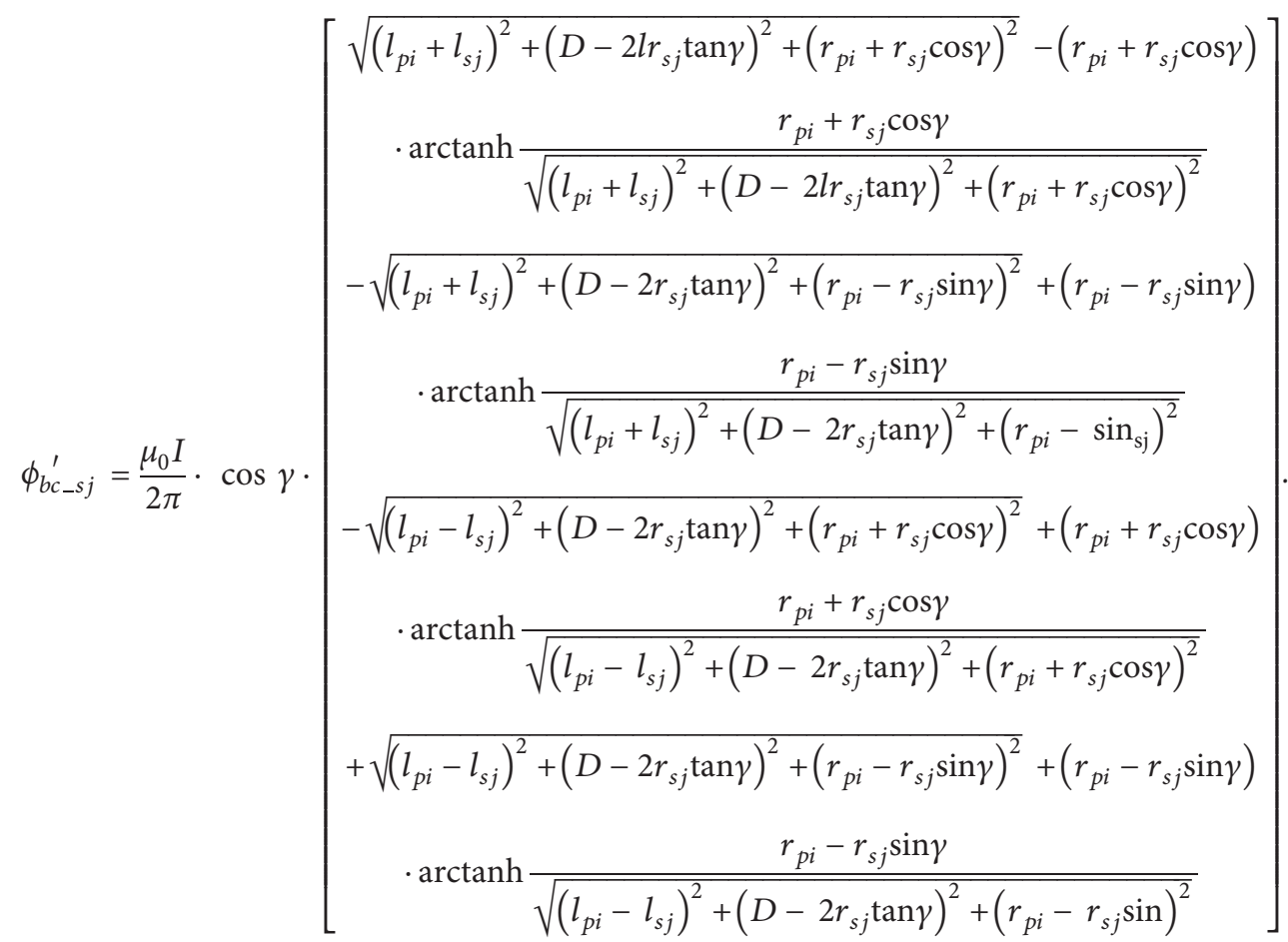

According to the same method, the magnetic fluxes of segments $\mathrm{cd}$, da, and ab in the secondary coil are obtained, respectively. According to equations (12) and (13), the mutual inductance value when the coil is tilted can be obtained, and the coupling coefficient of the coil in the inclined state can be obtained.

\section{Finite Element Simulation}

The construction and analysis of finite element simulation model is very complicated and time-consuming, but the calculation results are very close to the real values. Therefore, in order to verify the correctness of the mutual inductance model in the offset and tilt states, ANSYS Maxwell finite element simulation software is used to establish the coupling coil model, and the magnetic field distribution and coupling coefficient of different coupling coils are analyzed. The simulation value of coupling coefficient is compared with the theoretical value. The coil parameters used in the simulation model and theoretical calculation are shown in Table 1.

Figure 8 shows a comparison of magnetic field parallel to the coil when the coil is shifted or tilted. The underwater ICPT coil gap is usually less than $30 \mathrm{~mm}$. Here, the gap is set to $30 \mathrm{~mm}$ when the coils are parallel. In order to avoid contact between coils, the maximum tilt angle at this distance is set to $10^{\circ}$ according to the coil size parameter.

According to the magnetic field distribution in Figure 8, the magnetic field is symmetrically distributed on both sides of the coil when the coils are coaxial and parallel. The coil deviates or tilts, the magnetic field distribution changes obviously, and the magnetic field is in the asymmetric distribution of the secondary coil, which is the essential reason for the change of mutual inductance.

The parameter scanning function in ANSYS software is used to study the change of coupling coefficient under different coil states. First, the offset coefficient $g$ is defined as the ratio of the coil offset $\Delta$ to the side length of the first turn coil. When the coil is offset along the long or short side, its offset coefficient can be expressed as

$$
\left\{\begin{array}{l}
g_{x}=\frac{\Delta_{x}}{2 r_{s 1}}, \\
g_{y}=\frac{\Delta_{y}}{2 r_{s 1}} .
\end{array}\right.
$$

The offset coefficient is set as the scanning item, and the scanning step size is set to 0.05 . The change curve of the coupling coefficient of the coil is obtained when $g_{x}$ and $g_{y}$ change from -0.5 to 0.5 . The comparison between the simulation results and the theoretical calculation values is shown in Figure 9. It can be seen from the figure that the coupling coefficient will decrease with the increase of offset distance. When the offset coefficient is reached, the coupling coefficient is lower than 0.2 , and the coupling coefficient change rate can reach $65 \%$. According to this trend, it can be predicted that, with 


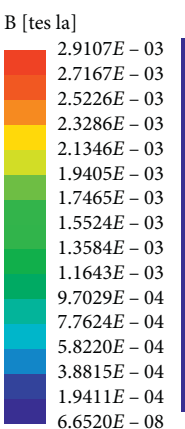

$6.6520 E-08$

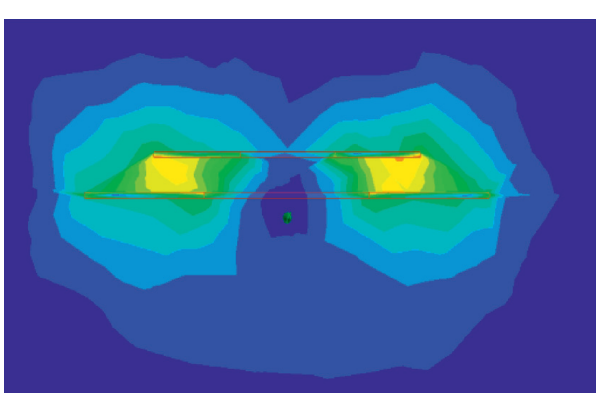

(a)
B [tes la]

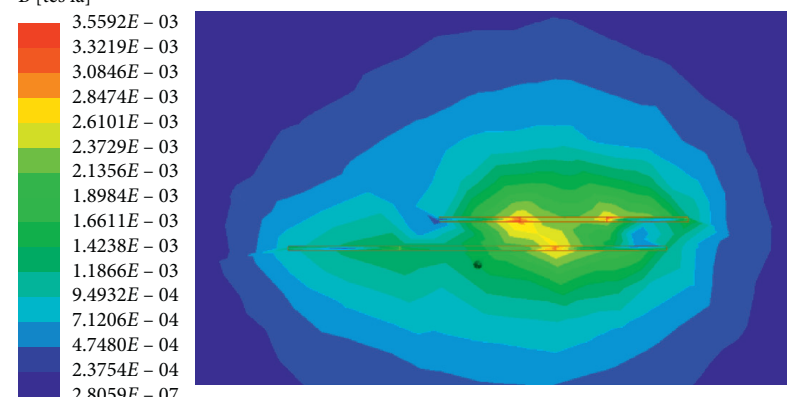

(b)

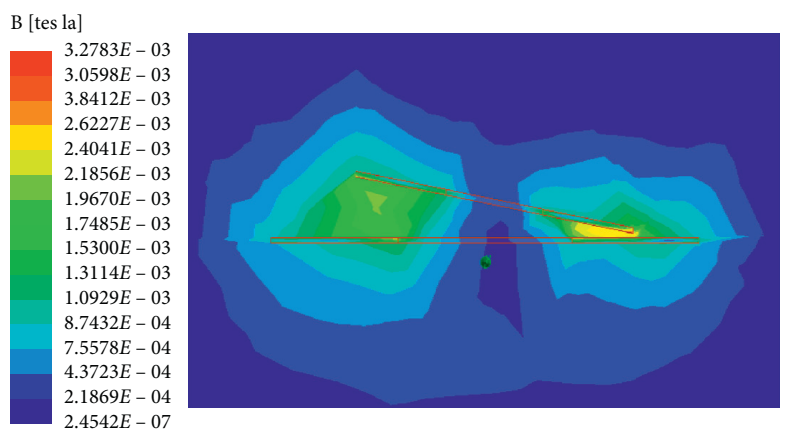

(c)

Figure 8: Magnetic field distribution of the coil at different relative positions. (a) Magnetic field distribution of the coil coaxial and parallel. (b) Magnetic field distribution of coil with horizontal offset of $100 \mathrm{~mm}$. (c) Magnetic field distribution of coil angle inclined $10^{\circ}$.

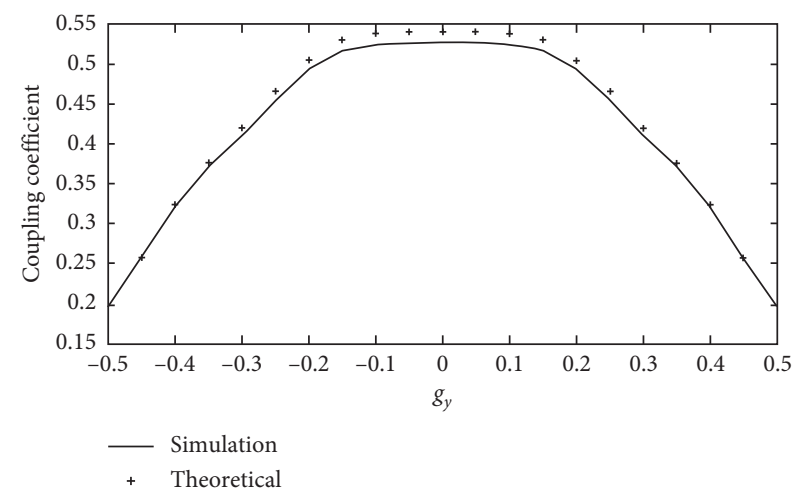

(a)

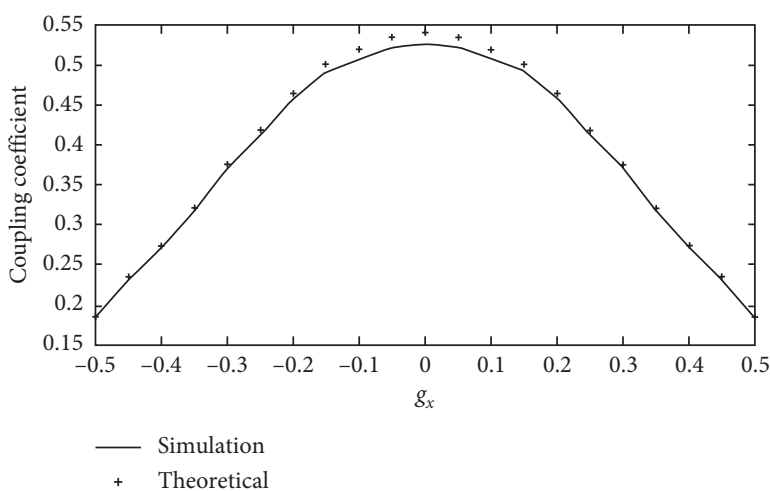

(b)

FIGURE 9: Coupling coefficient of horizontal offset of the secondary coil. (a) Offset along the long side. (b) Offset along the short side.

the further increase of migration distance, the coupling coefficient will gradually approach 0 and the transmission efficiency will tend to 0 . At the same time, when the coupling coefficient drops to 0.2 , the transmission efficiency will drop sharply.

Keeping other parameters unchanged, the tilt angle is set as the scanning term, and the scanning step size is set to $1^{\circ}$, and the coupling coefficient is obtained when the secondary coil is tilted $-10^{\circ}$ to $10^{\circ}$ along the $Y$ direction or $X$ direction. According to the information in Figure 10, the secondary coil is tilted $-10^{\circ}$ to $10^{\circ}$ and the change rate of coupling coefficient is less than $3 \%$, which means that the angle tilt within $10^{\circ}$ will not greatly affect the transmission efficiency of the system. Due to the limitation of the coil geometry, the inclination of $10^{\circ}$ has reached the tilt limit in the gap of $30 \mathrm{~mm}$. Therefore, the influence of angle tilt on transmission efficiency can be ignored in this gap.

\section{Experimental Verification}

In order to further verify the theoretical analysis of this article, an underwater ICPT experimental platform was set up to verify the mutual inductance model. 


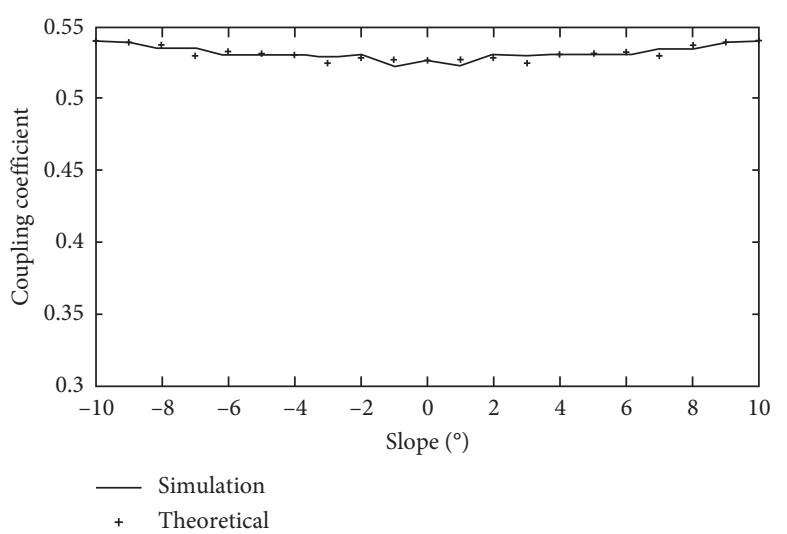

(a)

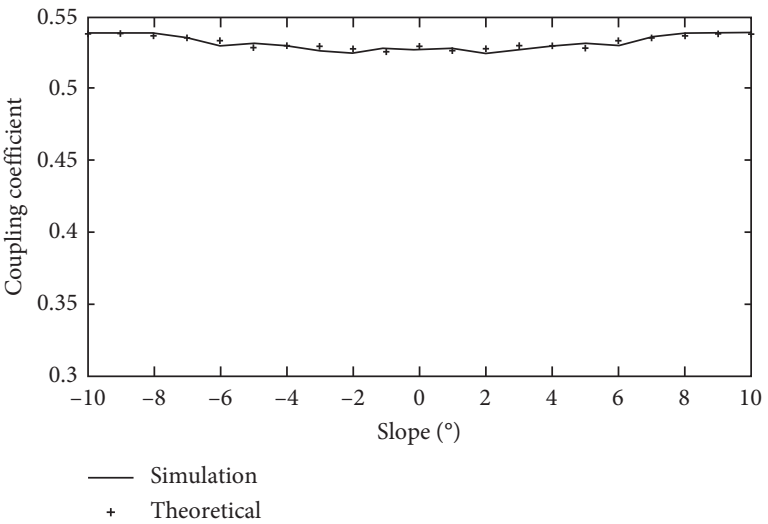

(b)

FIgURE 10: Coupling coefficient of secondary coil inclination angle less than $30 \mathrm{~mm}$. (a) Tilt in the $Y$ direction. (b) Tilt in the $X$ direction.

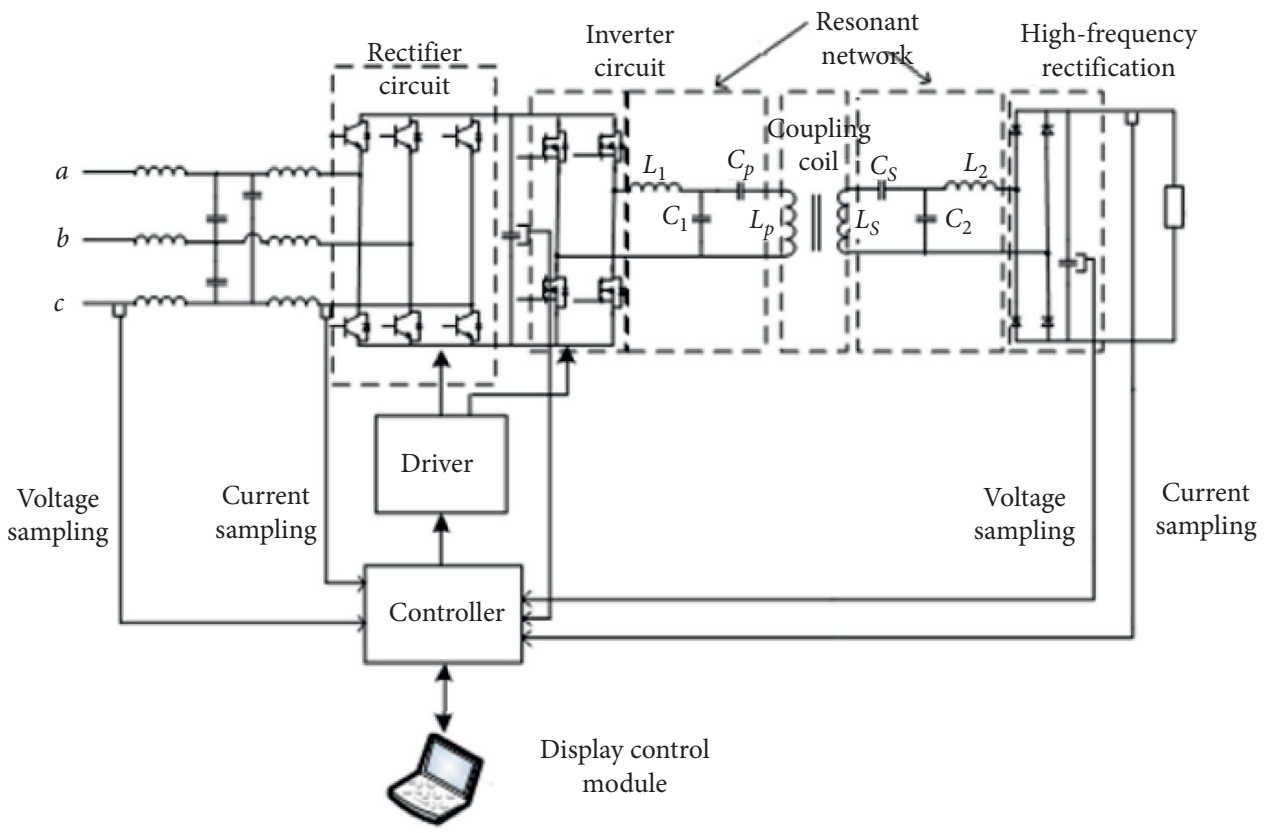

Figure 11: Underwater ICPT system.

4.1. Experiment Platform. The structure of the experimental platform is shown in Figure 11. In the primary side, the three-phase AC power is rectified to $85 \mathrm{khz}$ highfrequency $\mathrm{AC}$, and the obtained high-frequency $\mathrm{AC}$ is transmitted to the transmitting coil through the primary side resonant network. The coupling between them is transferred to the secondary side, then flows into the highfrequency rectifier module through the secondary side resonant network, and finally obtains the DC power required by the load.

The main controller in the system is mainly composed of sampling circuit and DSP. In this article, DSP uses TMS320F28335 chip. The voltage and current sampling is completed by Hall sensor. The rated output power of the system is $3.3 \mathrm{kw}$, and the load is composed of a power resistor with a total resistance of $30 \Omega$. MOSFET is used in the full bridge inverter circuit, and its model is SCT3040KL. The number of litz wires is 1300 , the single wire diameter is $0.1 \mathrm{~mm}$, the coil turns are $7.2 \mathrm{~mm}$, and the primary and secondary coil outer diameters are $230 \mathrm{~mm}$ and $180 \mathrm{~mm}$, respectively. The physical modules are shown in Figure 12. In order to prevent the coil from being immersed in the water, the transmission coil will not directly contact the seawater during the experiment. The transmitting and receiving coils are, respectively, placed at the bottom and top of the water injection tank to simulate the underwater transmission.

4.2. Mutual Inductance Model Verification. In order to verify the correctness of the coil mutual inductance model 

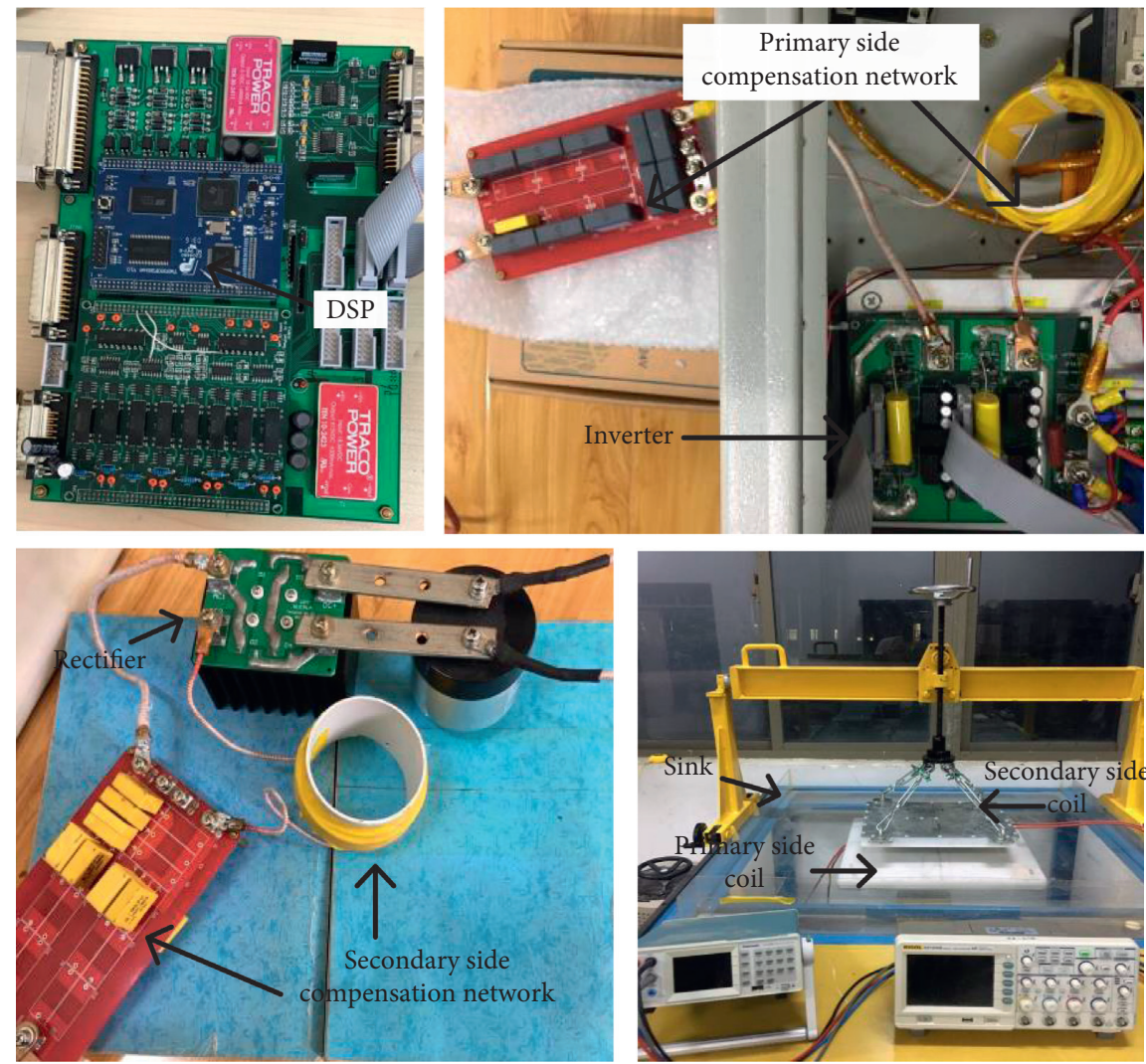

FIGURE 12: Underwater ICPT experimental platform.

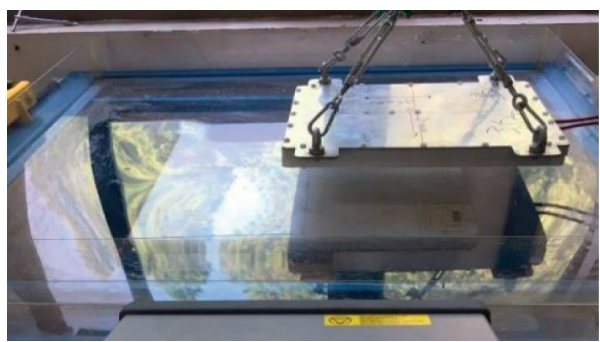

(a)

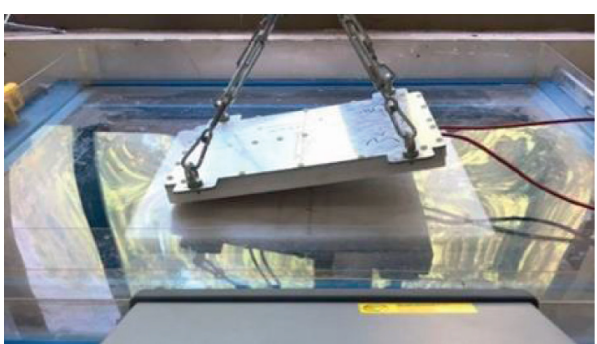

(b)

FIgURE 13: Coil setting in mutual inductance model verification experiment. (a) Migration experiment. (b) Tilt test.

proposed in bias or tilt state, an experimental platform is designed for verification experiments. The coil settings in the experiment are shown in Figure 13. In the experiment, the inductance parameters were measured by a Keysight E4980AL impedance analyzer.

In the experiment, the self-inductance of the coil can be measured directly. The measurement method of mutual inductance is as follows: connect the different ends of the coil in series, measure the inductance between the other two ports, and record it as $L^{+}$; the inductance between the two ports is recorded as $L^{-}$. According to the circuit Kirchhoff's law, the value of the mutual inductance $M$ can be obtained:

$$
M=\frac{L^{+}-L^{-}}{4} .
$$

The magnetic permeability of seawater and air is very close; the coupling coefficient of coils in the two media can be regarded as the same in the same space position. First, verify this conclusion and record the coupling coefficient data of the coil when the transmission gap changes from $30 \mathrm{~mm}$ to $150 \mathrm{~mm}$ under the media of air and seawater, as shown in Figure 14. The experimental results show that the difference between the two media is less than $1 \%$. Therefore, in order to simplify the experimental process, the following dislocation experiments use air medium instead of seawater medium. 


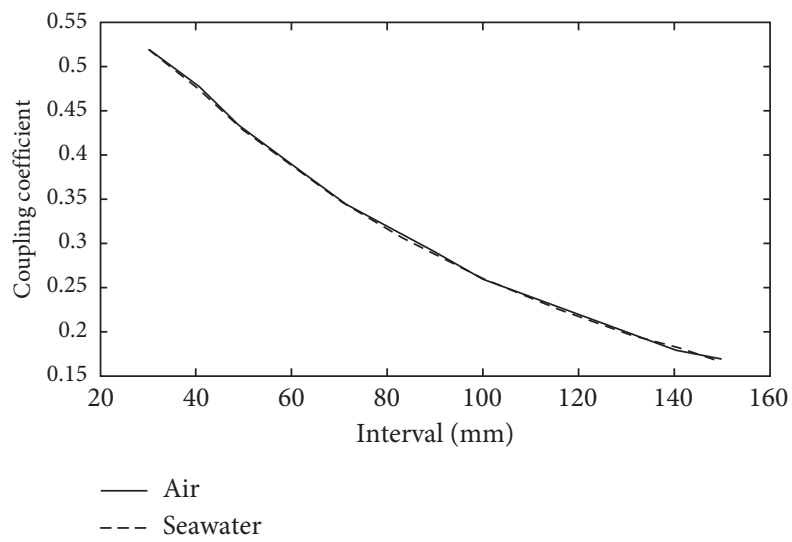

Figure 14: Comparison of coupling coefficients between air and seawater.

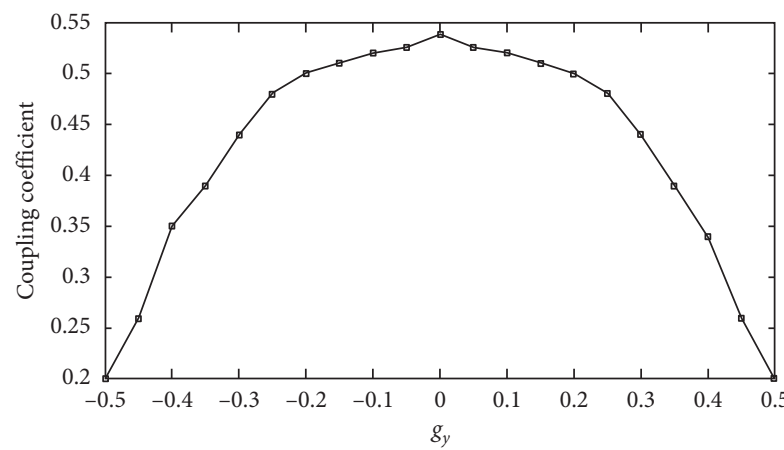

(a)

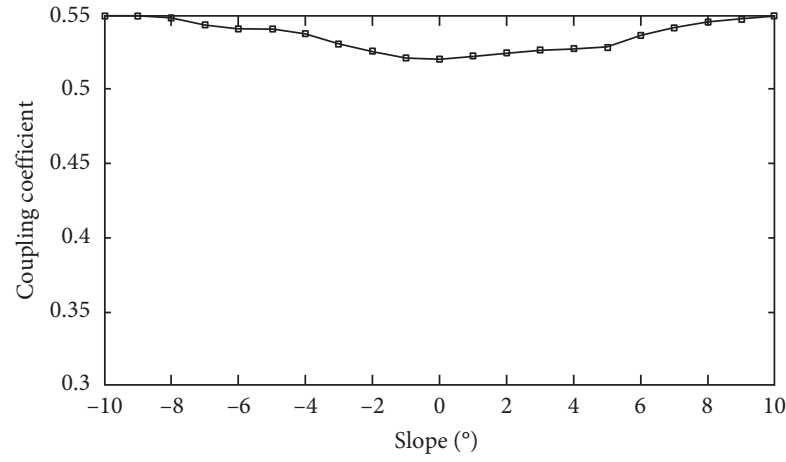

(c)

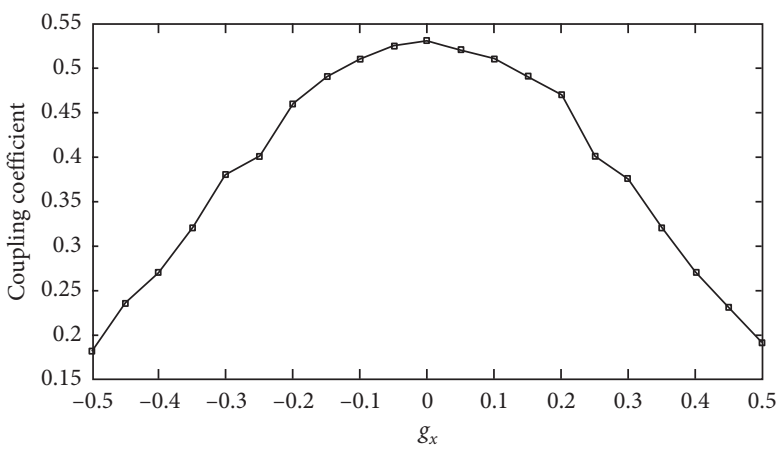

(b)

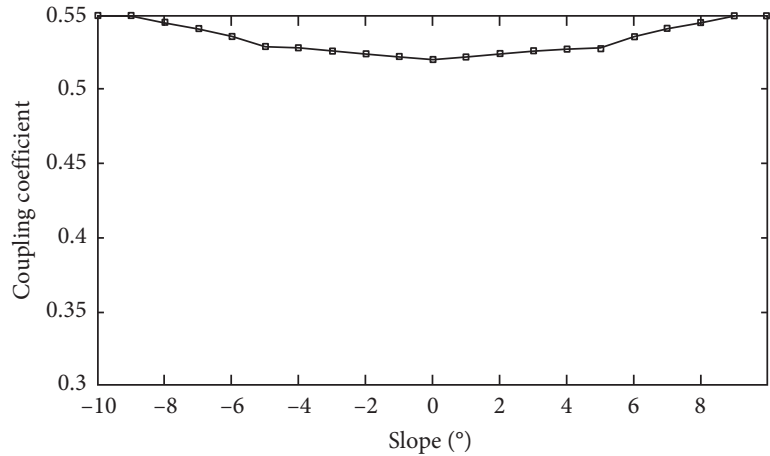

(d)

Figure 15: Coupling coefficient under misalignment of secondary coils with spacing of $30 \mathrm{~mm}$. (a) Offset along the long side. (b) Offset along the short side. (c) Tilt along the length. (d) Inclined in the width direction.

The coil gap length is fixed to $30 \mathrm{~mm}$. In the experiment, the variation range of secondary coil offset coefficient is set as $-0.5 \sim 0.5$, and the tilt range is $-10^{\circ} \sim 10^{\circ}$. The experimental results of offset and tilt are shown in Figure 15.

As can be seen from Figures 15(a) and 15(b), the coupling coefficient will decrease with the increase of migration distance. The offset coefficient reaches \pm 0.5 ; the coupling coefficient falls below 0.2. From Figures 15(c) and 15(d), it can be seen that the average change rate of the coupling coefficient is less than $4 \%$ when the coil is tilted by $-10^{\circ} \sim 10^{\circ}$.
The coupling coefficient is not affected when the coil tilts in this angle range.

4.3. Wireless Power Transmission in Different Media. In order to study the effect of different media on the transmission efficiency of ICPT, fresh water and seawater were injected into the gap tank to simulate different transmission environments. The seawater used in the experiment was obtained from the ratio of fresh water and sea salt, and the conductivity of the seawater was $4.5 \mathrm{~S} / \mathrm{m}$, and the 


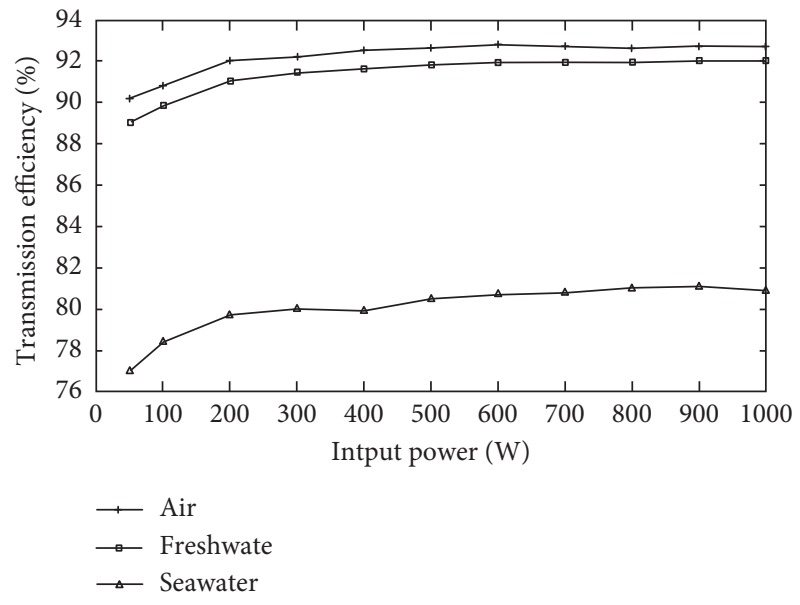

(a)

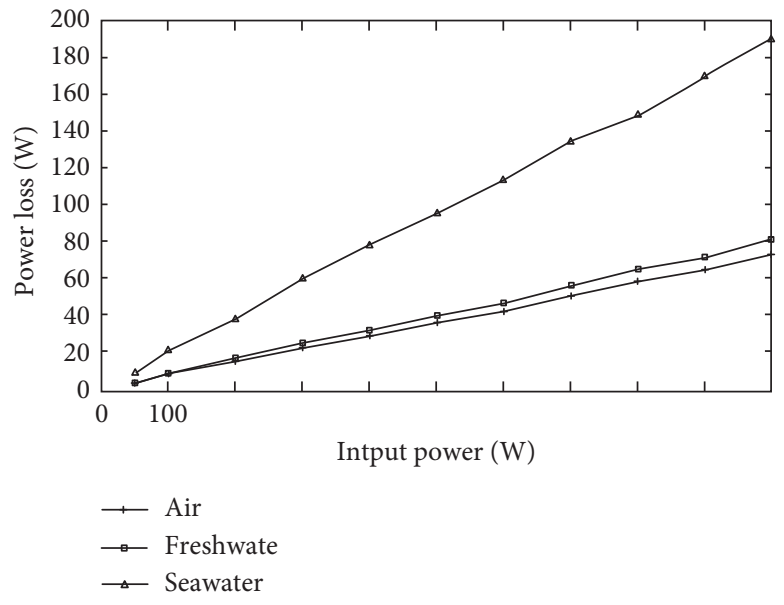

(b)

Figure 16: Transmission characteristics with fixed gap. (a) Transmission efficiency with fixed gap. (b) Power loss with fixed gap.

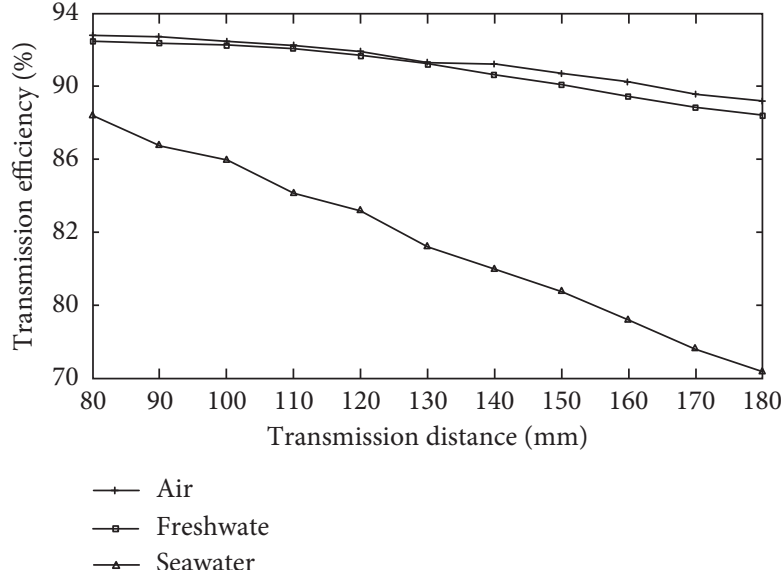

(a)

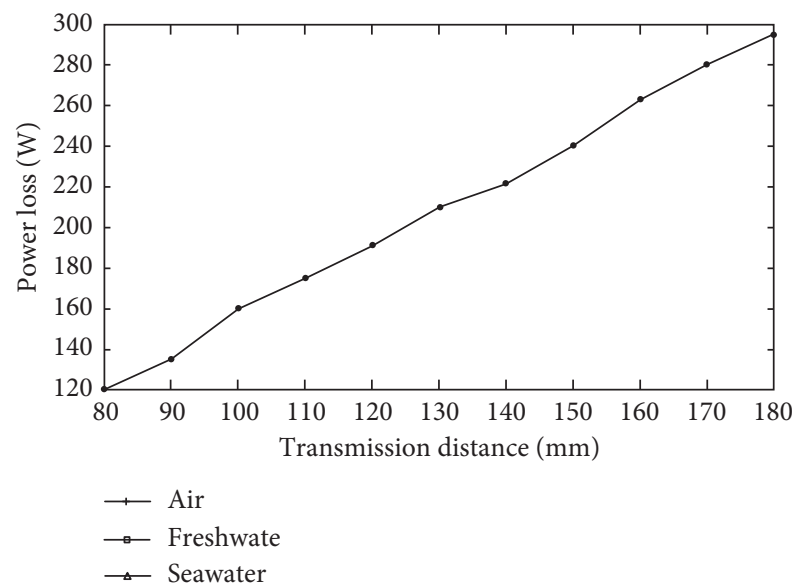

(b)

Figure 17: Transmission characteristics with fixed input power. (a) Transmission efficiency with fixed input power. (b) Power loss and transmission distance in seawater.

conductivity parameters were measured by ShengCi DDS11 conductivity tester. The transmission gap is fixed to $120 \mathrm{~mm}$; the transmission efficiency and power loss under different media are recorded and compared with the data under the air. The result is shown in Figure 16. The power and efficiency data are measured by the power analyzer of Yokogawa WT1800. Since it is difficult to directly measure the eddy current loss of the medium, the transmission loss in the seawater and freshwater medium in the experiment is the difference between the measured loss and the transmission loss of the air medium.

Due to the existence of eddy current loss in seawater, the transmission efficiency of ICPT in this environment is lower than that of air and freshwater, and the loss power is positively correlated with input power. Under $1 \mathrm{~kW}$ input power, the transmission efficiency in seawater medium is about $14 \%$. As the excitation voltage increases, the magnetic induction intensity generated by the excitation also increases. According to the eddy current loss calculation formula given in [19], the eddy current loss power is proportional to the square of the magnetic induction intensity.

The input power of primary side is fixed at $1000 \mathrm{~W}$, and the transmission efficiency data of ICPT in different media are recorded at the transmission distance of $80 \mathrm{~mm} \sim 180 \mathrm{~mm}$. The results are shown in Figure 17(a). The data of transmission loss changing with transmission distance in seawater medium is further recorded, as shown in Figure 17(b).

In air and fresh water media, with the increase of transmission distance $h$, the change rate of transmission efficiency is obviously lower than that of seawater medium. This is because the coupling coefficient of coil is greater than 0.2 in the range of this gap. 


\section{Conclusion}

In this paper, the effect of seawater as a special transmission medium on the transmission performance of ICPT is studied. The change of self-inductance and mutual inductance of coupling coil under seawater pressure is analyzed by using ANSYS electromagnetic simulation platform. In view of the fact that the coupling coil is easy to be out of alignment under the action of ocean current, the mutual inductance calculation model of the coil under bias and tilt state is analyzed, and the magnetic field distribution is simulated by using the simulation platform. The conclusions of this paper are helpful to the optimization design of coupling structure of ICPT system in marine environment.

(1) With the increase of the offset range, the coupling coefficient will gradually decrease, and the transmission efficiency of the system will decrease. At the gap of $30 \mathrm{~mm}$, the offset is less than half of the side length, and the transmission efficiency is relatively stable because the coupling coefficient is greater than 0.2. The offset is greater than one-half of the side length; the coupling coefficient reaches the efficiency change. If the offset is further increased, the transmission efficiency will decrease sharply.

(2) In air and fresh water medium, with the increase of transmission distance, the change rate of transmission efficiency is obviously lower than that of seawater. In the transmission range of $80 \mathrm{~mm}-180 \mathrm{~mm}$, the system has not reached the threshold value of efficiency mutation, so the efficiency decline is relatively gentle; due to the existence of eddy current loss, the change rate of efficiency can reach $21 \%$ at $180 \mathrm{~mm}$ distance in seawater. At the same time, the input power is fixed, the transmission loss is positively correlated with the transmission distance, and the relationship between them is linear.

\section{Data Availability}

The data used to support the findings of this study were supplied by DaLi under license and so cannot be made freely available. Requests for access to these data should be made to DaLi,957297910@qq.com.

\section{Conflicts of Interest}

The authors declare that they have no conflicts of interest.

\section{Acknowledgments}

This work was supported in part by the National Natural Science Foundation of China (51807197) and Innovation Group Talents Project of Hubei Natural Science Foundation (2018CFA008).

\section{References}

[1] X. Huang, L. Tan, and C. Zhong, "Summary of research and application of wireless energy transmission technology," Journal of Electrical Engineering and Technology, vol. 28, no. 10, pp. 1-11, 2013.
[2] W. Chen and Q. Chen, "Research progress and application of resonant wireless energy transmission technology," Advanced Technology of Electrical Engineering and Energy, vol. 35, no. 9, pp. 35-47, 2016.

[3] X. Fan, X. Mo, and X. Zhang, "Research status and application of wireless energy transmission technology," Proceedings of the CSEE, vol. 35, no. 10, pp. 2584-2600, 2015.

[4] Z. Zhao, Y. Zhang, and K. Chen, "New progress in magnetically coupled resonant wireless energy transmission technology," Proceedings of the CSEE, vol. 33, no. 3, pp. 1-13, 2013.

[5] H. Zhou, Y. Jiang, and W. Hu, "Research and review on the safety of electromagnetic environment in the application of magnetic resonance wireless power transmission system," Transactions of China Electrotechnical Society, vol. 31, no. 2, pp. 1-12, 2016.

[6] X. Wu, P. Sun, and S. Yang, "Summary of research on underwater radio energy transmission technology and application," Journal of Electrical Engineering and Technology, vol. 34, no. 8, 2019.

[7] Z. Gao, Y. Li, and Q. Jing, "Coupling mechanism of electric field coupling underwater wireless energy transmission system," Journal of Hohai University (Natural Sciences), vol. 46, no. 4, pp. 366-370, 2018.

[8] G. Tao, X. Liu, and Y. Wang, "Research on the influence of electric vehicle wireless charging system parameters on coupling," Journal of Jilin University (Information Science Edition), vol. 2, no. 6, pp. 55-61, 2017.

[9] H. Zhou, Research on LCL Resonant Inductive Coupling Power Transmission System, Shanghai Jiaotong University, Shanghai, China, 2014.

[10] J. Gao, X. Wu, and Y. Gao, "Dynamic voltage regulation control method of electromagnetic induction non-contact power transmission system based on LCL resonant converter," Journal of Electrical Engineering and Technology, vol. 33, no. 18, pp. 31-40, 2018.

[11] Y. Guo, C. Li, and N. Cui, "Analysis and control of constant current output based on LCL-S type ICPT system," Journal of Power Sources, vol. 34, no. 2, 2017.

[12] W. Ni, Q. Yang, and L. Yang, "Influence of lateral misalignment of inductive devices on coupling coefficients in wireless power transmission systems," Journal of Tianjin Polytechnic University, vol. 1, no. 2, pp. 45-47, 2014.

[13] M. Kiani and M. Ghovanloo, "The circuit theory behind coupled-mode magnetic resonance-based wireless power transmission," IEEE Transactions on Circuits and Systems I: Regular Papers, vol. 59, no. 9, Article ID 2065, 2012.

[14] Z. He, "Research on three-dimensional omnidirectional underwater wireless energy transmission method," Harbin Institute of Technology, Harbin, Heilongjiang. China, 2017.

[15] K. Zhang, L. Yan, and Z. Yan, "Modeling and loss analysis of underwater non-contact power transmission system based on magnetic resonance," Acta Physica Sinica, vol. 65, no. 4, 2016.

[16] A. Askari, R. Stark, and J. Curran, "Underwater wireless power transfer," in Proceedings of the Wireless Power Transfer Conference, May 2015.

[17] K. Shizuno, S. Yoshida, and M. Tanomura, "Long distance high efficient underwater wireless charging system using dielectric-assist antenna," in Proceedings of the Oceans, St. John's, NL, Canada, September 2015.

[18] J.-S. Gao and N. Zhang, "Giant piezomagnetism and piezoimpedance effects in MnZn ferrite device under hydrostatic pressure," Journal of Inorganic Materials, vol. 24, no. 1, pp. 87-90, 2009.

[19] J. Zhou, Research on Optimization of Non-Contact Power Transmission Efficiency in Seawater Environment, Zhejiang University, Hangzhou, China, 2014. 